\title{
Throughput Analysis of ETSI BRAN HIPERLAN/2 MAC Protocol Taking Guard Timing Spaces into Consideration
}

\author{
You-Chang $\mathrm{Ko}^{1}$, Eui-Seok Hwang ${ }^{2}$, Jeong-Shik Dong ${ }^{2}$, Hyong-Woo Lee ${ }^{3}$, and \\ Choong-Ho $\mathrm{Cho}^{2}$ \\ ${ }^{1}$ LG Electronics Inc. UMTS Handsets Lab, \\ Korea \\ ycko@lge.com \\ ${ }^{2}$ Dept. of Computer Science \& Technology, \\ KOREA UNIV., Korea \\ \{ushwang, bwind, chcho\} @korea.ac.kr \\ ${ }^{3}$ Dept. of Electronics \& Information Engineering, \\ KOREA UNIV., Korea \\ hwlee@korea.ac.kr
}

\begin{abstract}
In this paper we examine the effects of the required portions of guard timing spaces in a MAC frame of ETSI BRAN HIPERLAN/2 system such as inter-mobile guard timing space in UL(Up Link) duration, inter-RCH(Random CHannel) guard timing space, sector switch guard timing space. In particular, we calculate the number of OFDM(Orthogonal Frequency Division Multiplexing) symbols required for these guard timing spaces in a MAC frame. We then evaluate the throughput of HIPERLAN/2 system as we vary parameter such as the guard time values defined in [2], the number of DLCCs(Data Link Control Connections), and the number of RCHs. Finally we show by numerical results that the portions for the total summation of required guard timing spaces in a MAC frame are not negligible, and that they should be properly considered when trying to evaluate the performance of MAC protocol of HIPERLAN/2 system and also when determining the number of RCHs as well as the number of DLCCs in UL PDU trains at an AP/CC(Access Point/Central Controller).
\end{abstract}

\section{Introduction}

HIPERLAN/2, providing high-speed communications between mobile terminal and various broadband infrastructure networks and representing a centralized access of MAC protocol, is an ETSI BRAN standard with a $2 \mathrm{~ms}$ duration of MAC frame. There have been studies which analyze throughput of HIPERLAN/2 MAC layer[4][5]. Their first step to analyze the throughput of MAC layer is to calculate the length of the LCHs(Long transmit CHannels) in a number of OFDM symbols in a MAC frame in such a manner that the overhead durations(preambles and signals) for each channels and PDU trains are subtracted from the whole duration of a MAC frame. In these studies, however, they omit to subtract from the total length of a MAC frame some of 
non-negligible components of overheads in a MAC. Firstly, they do not take into account some guard timing spaces in order to cope with propagation delay such as inter-mobile guard timing space in UL duration, inter- $\mathrm{RCH}$ guard timing space, and sector switch guard timing space which is needed in a multi-sector environment. Secondly, they omit to subtract the overhead portions of DL(Down Link) PDU train as well as $\mathrm{SCHs}$ (Shot transmit CHannels) of UL PDU train from a MAC frame duration. Because of these reasons, the previous works tend to overestimate the possible length of LCHs as the pure user data path, and consequently may lead to inflating result of its throughput. Having considered the missing points of the previous works and further taken into account multi-sector environment and DiL(Direct Link) PDU train as optional, what we try to show in this paper is the effects on the throughput of the required guard timing spaces imposed on a MAC frame based on [1][2]. Specifically we observe the results as varying the number of active user's DLCC as well as guard time values defined in [2]. Finally we show by numerical results that the whole required portions of the guard timing spaces are not negligible within a MAC frame duration of 500 OFDM symbols, as the number DLCC and RCH is increased. These overheads should be properly considered when evaluating the performance of HIPERLAN/2 system, and also when determining the number of RCHs as well as the number of DLCCs in UL PDU trains at an AP/CC.

In section II we introduce system parameters to evaluate throughput of HIPERLAN/2 system in this paper. In section III we analyze the length of each PDU parts regarding guard timing spaces for throughput analysis in a MAC frame. Section IV deals with various numerical results examining the effects of the number of DLCCs as well as guard time values. Finally we end with conclusions in section V.

\section{System Parameters}

The PHY(physical) layer of HIPERLAN/2 is based on the modulation scheme OFDM with 52 sub-carriers whose possible modulations are BPSK(Binary Phase Shift Keying), QPSK(Quaternary Phase Shift Keying), and 16QAM(Quadrature Amplitude Modulation) for mandatory, and 64QAM for optional. In order to improve radio link capacity due to different interference situations and distances of MTs to the AP or CC, a multi-rate PHY layer is applied, where the appropriate mode can be selected by the link adaptation scheme. The mode dependent parameters are listed in TABLE I. We assume that the preambles for UL PDU trains and RCH PDUs are long preamble i.e. four OFDM symbols and each active user has two DLCCs; one is for UL and the other is for DL. We further assume that every DLCC has one SCH, which is only used to convey control user data, and also that the guard time value for $\mathrm{RCH}$ specified in $\mathrm{BCCH}$ (Broadcast Control CHannel) is concurrently applied to that for UL interPDU trains. We define and assume system parameters for numerical calculation as TABLE II. 
Table 1. Phy layer mode dependent parameters

\begin{tabular}{cccc}
\hline Modulation & $\begin{array}{c}\text { Coding } \\
\text { rate }\end{array}$ & $\begin{array}{c}\text { Nominal bit } \\
\text { rate[Mbps] }\end{array}$ & $\begin{array}{c}\text { Data bits/octets } \\
\text { per OFDM } \\
\text { symbol }\end{array}$ \\
BPSK & $1 / 2$ & 6 & $23 / 3$ \\
BPSK & $3 / 4$ & 9 & $36 / 4.5$ \\
QPSK & $1 / 2$ & 12 & $48 / 6$ \\
QPSK & $3 / 4$ & 18 & $72 / 9$ \\
16QAM & $9 / 16$ & 27 & $108 / 13.5$ \\
16QAM & $3 / 4$ & 36 & $144 / 18$ \\
64QAM & $3 / 4$ & 54 & $216 / 27$ \\
\hline
\end{tabular}

Table 2. Parameters for numerical calculations

\begin{tabular}{|c|c|c|}
\hline Parameters & Meaning & Value \\
\hline Lx & The length of X PDU in a MAC frame. & Variable \\
\hline$B p S x$ & $\begin{array}{l}\text { The number of bytes coded per OFDM symbols } \\
\text { for X PDU train. }\end{array}$ & $\begin{array}{l}\text { Depend on PHY } \\
\text { layer modes }\end{array}$ \\
\hline $\mathrm{N}_{\mathrm{sec}}$ & The number of sectors per AP. & $1 \sim 8$ \\
\hline $\mathrm{NIE}$ & The number of IE blocks in whole sectors. & Variable \\
\hline $\mathrm{NX} X_{\mathrm{SCH}}$ & The total number of $\mathrm{SCH}$ in $\mathrm{X}$ PDU train. & 1 \\
\hline NXMT & The number of active MTs in X PDU train. & Variable \\
\hline NDiL_MT_Diff & $\begin{array}{l}\text { The number of different index of transmitter } \\
\text { between two consecutive MTs in DiL PDU train. }\end{array}$ & $\begin{array}{l}\text { Variable } \\
\text { (Optional) }\end{array}$ \\
\hline $\mathrm{S}_{\mathrm{g}}$ & Sector switch guard time & $800 \mathrm{~ns}$ \\
\hline $\mathrm{Pg}$ & Propagation delay guard time & $2.0 \mu \mathrm{s} \sim 12 \mu \mathrm{s}$ \\
\hline UDoFDM & Unit Duration of an OFDM symbol & $4 \mu \mathrm{s}$ \\
\hline$\Delta(\mathrm{t})$ & Delta step function & $\begin{array}{l}0, t \leq 1 \text { and } \\
t, \text { elsewhere }\end{array}$ \\
\hline
\end{tabular}

\section{Throughput Analysis}

\subsection{BCH PDU Trains Length}

The $\mathrm{BCH}$ (Broadcast CHannel) has the size of 15 octets long and shall be transmitted using BPSK with coding rate $1 / 2$. The size of preamble shall consist of four OFDM symbols $(16 \mu \mathrm{s})$. According to Fig.1 we can calculate the length of BCH PDU train as (1) where sector switch guard timing space should be imposed on every interval between two BCHs if multi-sector is used. No sector switch guard timing space is inserted if omni-sector is used.

In the case of multi-sector, the total length of sector switch guard timing spaces in a number of OFDM symbols is calculated by dividing the sum of the number of guard times by $\mathrm{UD}_{\text {OFDM }}$. 


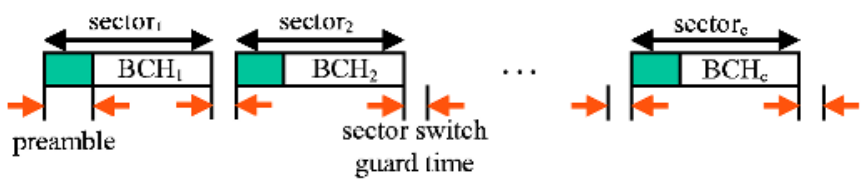

Fig. 1. The structure of BCH PDU trains

$$
\mathrm{L}_{\mathrm{BCH}}=\left(\mathrm{PRE}_{\mathrm{BCH}}+15 \text { bytes } / 3\right) * \mathrm{~N}_{\mathrm{sec}}+\frac{\Delta\left(N_{\mathrm{sec}}\right) \cdot S_{g}}{U D_{\text {OFDM }}}=9 * \mathrm{~N}_{\mathrm{sec}}+\frac{\Delta\left(N_{\mathrm{sec}}\right) \cdot S_{g}}{U D_{\text {OFDM }}}
$$

\subsection{FCH+ACH PDU Trains Length}

A FCH(Frame CHannel) shall be built of fixed size IE(Information Element) blocks. Every IE block shall contain three IEs, each with a length of eight octets and a CRC(Cycle Redundancy Check) of length 24 bits which shall be calculated over the three consecutive IEs.

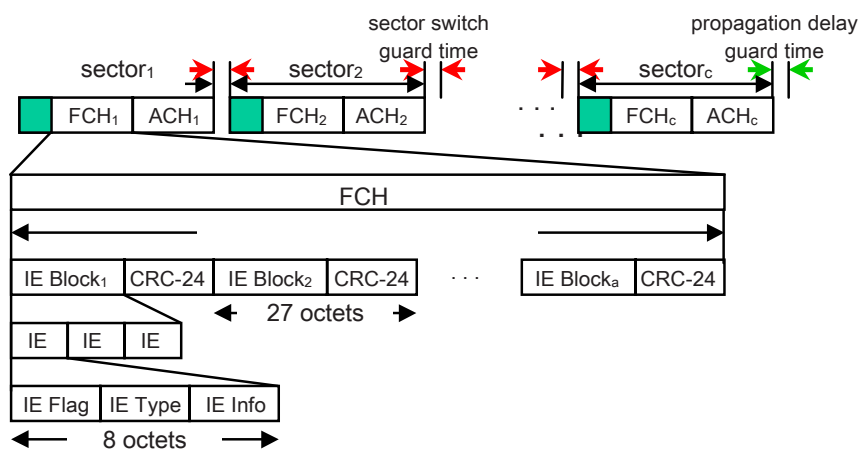

Fig. 2. The structure of $\mathrm{FCH}+\mathrm{ACH} P D U$ trains

Thus a FCH shall consist of multiple of 27 octets as Fig.2 depicts. The length of $\mathrm{ACH}$ (Access feedback CHannel) consists of nine octets. One preamble shall be added in the beginning of each $\mathrm{FCH}+\mathrm{ACH}$ PDU train if multiple sectors are used in AP and its size shall be two OFDM symbols. We assume one IE carries the signal information for one DLCC. Equation (2) shows the length of the FCH+ACH PDU train in a MAC frame. In the same fashion as in BCH PDU train, the sector switch guard timing space is set on every interval between $\mathrm{FCH}+\mathrm{ACH}$ PDUs, and the propagation delay guard timing space is also added at the end of the $\mathrm{FCH}+\mathrm{ACH}$ PDU train. If omni-sector is used only one preamble, size of four OFDM symbols, shall be imposed on the 
$\mathrm{BCH}+\mathrm{FCH}+\mathrm{ACH}$ train without any additional ticks. FCHs and ACHs shall use BPSK with coding rate $1 / 2$.

$$
\begin{aligned}
& \mathrm{L}_{\mathrm{FCH}+\mathrm{ACH}}=2^{*} \Delta\left(\mathrm{N}_{\mathrm{sec}}\right)+9^{*}\left\lceil\frac{N_{I E} \cdot 8}{24}\right\rceil+3^{*} \mathrm{~N}_{\mathrm{sec}}+\frac{\left(N_{\mathrm{sec}}-1\right) \cdot S_{g}+P_{g}}{U D_{\mathrm{DFDM}}}
\end{aligned}
$$

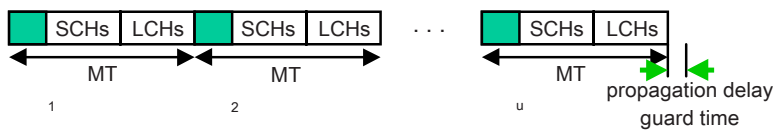

Fig. 3. The structure of DL PDU trains

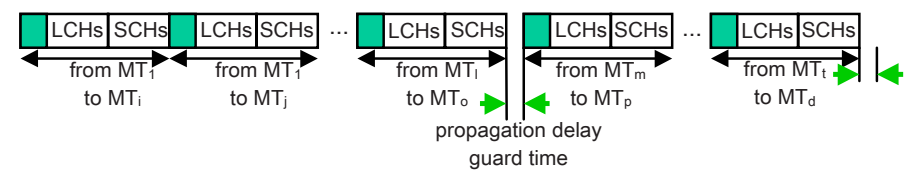

Fig. 4. The structure of DiL PDU trains

\subsection{PDU Trains Length}

Fig. 3 shows that the preamble, two OFDM symbols, is preceded with every first PDU, distinguished by DLCC-ID, in same MAC-ID, i.e. each MAC-ID GROUP, which consists of different DLCC-ID PDUs has only one preamble. One guard time shall be added at the end of the DL PDU train. Equation (3) shows the length of the DL PDU train except for LCHs in this PDU train.

$$
\mathrm{LDL-LCH}=2 * \mathrm{NDL}_{\mathrm{MT}}+\left\lceil\frac{9}{B p S_{S C H}}\right\rceil * \mathrm{NDLSCH}^{+} \frac{P_{g}}{U D_{O F D M}}
$$

\subsection{DiL PDU Trains Length as Optional}

Fig.4 shows that a guard timing space is needed where different TX mobile-id is positioned between two consecutive PDU trains.

Equation (4) shows the length of DiL PDU train except for LCHs in this PDU train.

$$
\mathrm{LDiL-LCH}=4{ }^{*} \mathrm{NDiL}_{\mathrm{MT}}+\left\lceil\frac{9}{B p S_{S C H}}\right\rceil{ }^{*} \mathrm{NDiLsCH}+\frac{\left(N D i L_{M T-D i f f}+1\right) \cdot P_{g}}{U D_{O F D M}}
$$




\subsection{UL PDU Trains Length}

Being different from DL PDU trains, UL PDU train always needs a guard timing space between two PDU trains whose MAC-IDs are different each other as in Fig.5. Equation (5) depicts the length of the UL PDU train except for LCHs duration. The guard timing spaces are directly proportional to the number of DLCCs used by active users in UL PDU trains as (5) shows.

$$
\text { LUL-LCH }=4 *{ }^{*} L_{M T}+\left[\frac{9}{B p S_{S C H}}\right] * \mathrm{NULSCH}+\frac{\Delta\left(N U L_{M T}\right) \cdot P_{g}}{U D_{O F D M}}
$$

Fig. 5. The structure of UL PDU trains

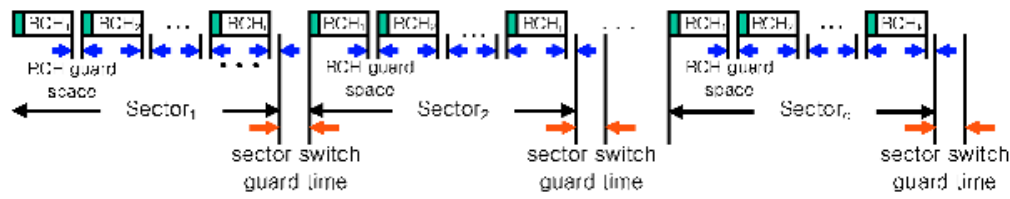

Fig. 6. The structure of RCH PDUs

\subsection{RCH PDUs Length}

At least one RCH shall be allocated per sector in each MAC frame and the RCHs for all sectors shall be clustered together. Between RCHs shall be space for preamble and

guard timing space as in Fig.6. The RCH consists of nine octets and its format is identical to that of the $\mathrm{SCH}$. The RCHs shall use BPSK with coding rate $1 / 2$. The size of RCH's preamble is same as that of UL, i.e. four OFDM symbols for long preamble or three OFDM symbols for short one. Equation (6) gives the total length of RCHs.

$$
\mathrm{LRCH}=7^{*} \mathrm{~N}_{\mathrm{RCH}}+\frac{N_{R C H} \cdot P_{g}+\Delta\left(N_{\mathrm{sec}}\right) \cdot S_{g}}{U D_{\text {OFDM }}}
$$

\subsection{Total Required Guard Timing Spaces and Maximum Throughput in a MAC Frame}

Using equations from (1) to (6), we can specify their durations into two parts where the first is the total length for the preambles and the signaling overheads $\left(\mathrm{L}_{\mathrm{PRE}+\mathrm{SOH}}\right)$, and the second is the total length for the guard timing spaces $\left(\mathrm{L}_{\mathrm{GTS}}\right)$. Subtracting $\mathrm{L}_{\mathrm{PRE}+\mathrm{SOH}}$ 
and $\mathrm{L}_{\mathrm{GTS}}$ from the total length of a MAC frame $\left(\mathrm{L}_{\mathrm{MF}}\right)$ in a number of OFDM symbols, we can now calculate the total length of LCHs for DL, DiL, and UL PDU trains in a MAC frame as (9).

$$
\begin{aligned}
& \mathrm{LPRE}_{\mathrm{PSOH}}=12{ }^{*} \mathrm{~N}_{\mathrm{sec}}+2^{*} \Delta\left(\mathrm{N}_{\mathrm{sec}}\right)+9 *\left\lceil\frac{N_{I E} \cdot 8}{24}\right\rceil+2^{*}\left(\mathrm{NDL}_{\mathrm{MT}}+2^{*} \mathrm{NDiLMT}+\right. \\
& \left.2{ }^{*} \mathrm{NUL}_{\mathrm{MT}}\right)+\left\lceil\frac{9}{B p S_{S C H}}\right\rceil *(\mathrm{NDLSCH}+\mathrm{NDiLsCH}+\mathrm{NULSCH})+7{ }^{*} \mathrm{~N}_{\mathrm{RCH}} \\
& \mathrm{LGTS}_{\mathrm{G}}=\left\lceil\frac{\left\{2 \cdot \Delta\left(N_{\mathrm{sec}}\right)+N_{\mathrm{sec}}-1\right\} \cdot S_{g}+\left\{3+N D i L_{M T_{-} D i f f}+\Delta\left(N U L_{M T}\right)+N_{R C H}\right\} \cdot P_{g}}{U D_{\text {OFDM }}}\right\rceil \\
& \mathrm{LLCH}_{\mathrm{LCH}}=\mathrm{L}_{\mathrm{MF}}-\left(\mathrm{L}_{\mathrm{PRE}}+\mathrm{SOH}+\mathrm{L}_{\mathrm{GTS}}\right)
\end{aligned}
$$

The LCH consists of 54 octets and shall be transported by using any modulation scheme in Table 1. Here one of our main concerns is $\mathrm{L}_{\mathrm{GTS}}$ imposed on MAC frame duration and its effects will be discussed in section IV. In order to get maximum throughput we use (10) as given in [4]. The total number of PDUs for $\mathrm{LCHs}\left(\mathrm{NPDU}_{\mathrm{LCH}}\right)$ per MAC frame is as follows :

$$
\mathrm{NPDU}_{\mathrm{LCH}}=\left\lfloor\frac{L_{L C H} \cdot B p S_{L C H}}{54}\right\rfloor
$$

Then the maximum throughput $\left(\mathrm{TP}_{\max }\right)$ is mainly influenced by $\mathrm{CL}$ (Convergence Layer) and is finally given by

$$
\mathrm{TP}_{\max }=N P D U_{L C H} \cdot \frac{x}{\left\lceil\frac{x+4}{48}\right\rceil} \cdot \frac{8}{t_{\text {frame }}}
$$

where $x$ is the length of the user data packets in bytes.

\section{Numerical Results}

In this section we examine the effects on the total required guard timing spaces and the maximum throughput of the number of RCHs, the number of DLCCs, and guard time values under various modulation schemes of HIPERLAN/2 system. In Fig.7, we observe the effect of the sector switch guard timing space and inter-RCH guard timing space by increasing the number of sectors whose maximum value is eight as specified in $\mathrm{BCCH}$. We assume that one RCH is included in each sector. Except for the case that maximum guard time value $(12 \mu \mathrm{s})$ is used, at most 13 OFDM symbols are required to carry the whole guard timing spaces even if the maximum number of sectors is hired. Referring to (8) we can see more clearly the effect of guard timing spaces required to support RCHs and $\mathrm{NUL}_{\mathrm{MT}}$ as shown in Fig.8. We assumed that $\mathrm{NDiL}_{\text {MT_Diff }}=0$ for the experiments in this paper, as it is optional. 


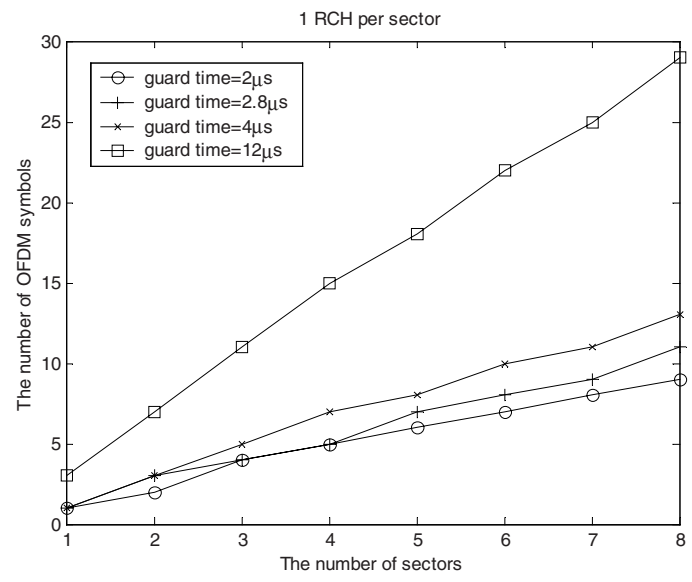

Fig. 7. The number of OFDM symbols by $\mathrm{L}_{\mathrm{GTS}}$ for $\mathrm{N}_{\mathrm{sec}} \mathrm{s}$

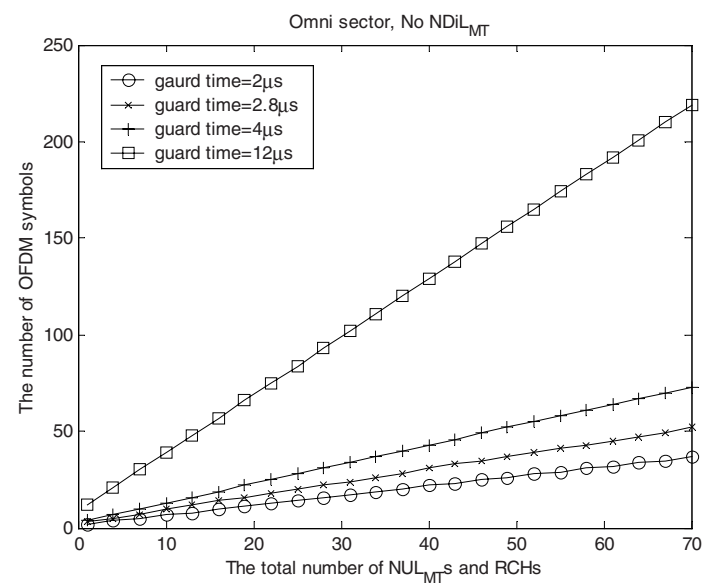

Fig. 8. The number of OFDM symbols by $\mathrm{L}_{\mathrm{GTS}}$ for $\mathrm{NUL}_{\mathrm{MT}} \mathrm{s}$ and RCHs

When the sum of number becomes 70, then the required number of OFDM symbols to carry their total guard timing spaces is 37 for minimum guard time value $(2 \mu \mathrm{s})$, and 219 for maximum guard time value $(12 \mu \mathrm{s})$. Increasing the number of active users in the system we have plotted the total required number of OFDM symbols according to (7), (8) in Fig.9. We can realize the differences of how many additional number of OFDM symbols there are needed when various guard time values defined in [2] are adapted. Under the assumptions and parameters we have made in Section II, when the number of active users reaches 20 the required number of OFDM symbols is over the total size of a MAC frame for the case that maximum guard time value is used. By changing the assumptions, especially adapting the use of SCH more sporadically, maximum active users can be increased in a MAC frame or the required number of OFDM symbols used by $\mathrm{L}_{\mathrm{PRE}+\mathrm{SCH}}$ and $\mathrm{L}_{\mathrm{GTS}}$ could be reduced. However the differences 
between the case of no guard time value and the cases of various guard time values are mainly influenced not by the assumptions but by guard time values, and its maximum difference is almost 100 OFDM symbols that is $20 \%$ of resources in a MAC frame. In Fig. 8 and Fig.9 we can see the differences that are becoming bigger by increasing the number of active users. This is owing to the fact that the total required guard timing spaces are directly proportional to the number of UL DLCCs used by active users as well as the allowed number of RCHs in the system. Fig.10 compares the maximum throughputs over the length of user data packet by the maximum modulation schemes(64QAM with coding rate 3/4), the minimum modulation scheme(BPSK with coding rate 1/2) and various guard time values.

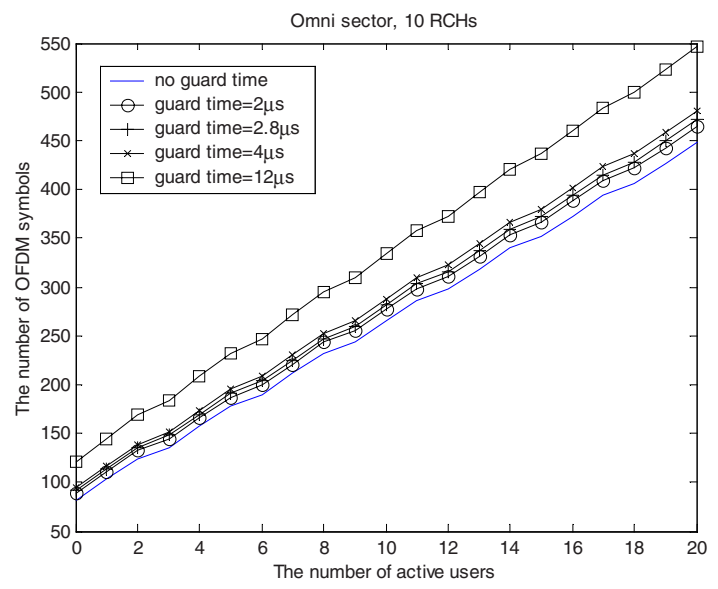

Fig. 9. The number of OFDM symbols by $\mathrm{L}_{\mathrm{PRE}+\mathrm{SCH}}$ and $\mathrm{L}_{\mathrm{GTS}}$

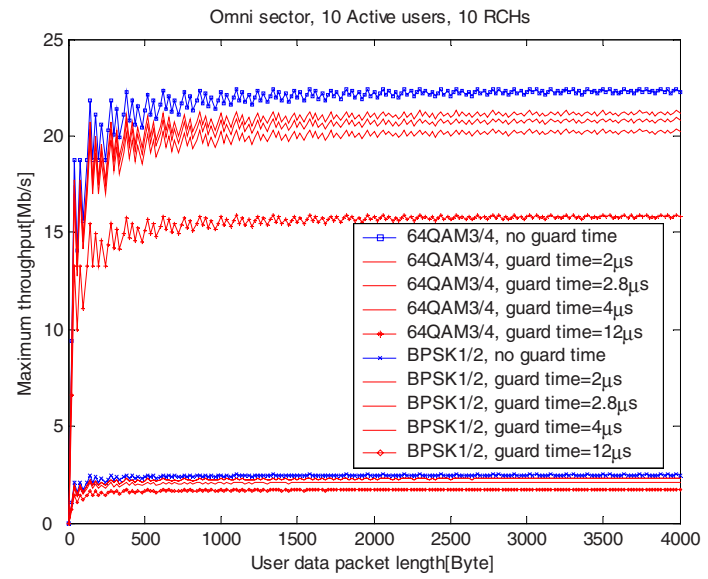

Fig. 10. Throughput by modulations and guard time values 


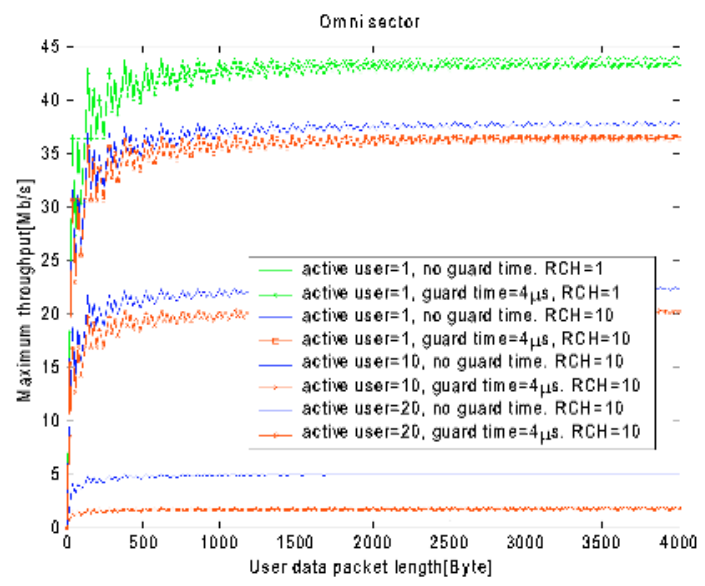

Fig. 11. Throughputs by 64QAM3/4 modulation for $\mathrm{LCH}$

We can see that the system throughputs are seriously degraded in comparison to the case with no guard time value in both maximum and minimum modulations as the guard time value has increased. The graphs of BPSK with coding rate $1 / 2$ for $2 \mu \mathrm{s}$ guard time value and BPSK with coding rate $1 / 2$ for $2.8 \mu$ s guard time value are completely overlapped with the third curve from the last one due to the property of floor function in (10). Fig.11 shows the throughput performances under the various number of active users and the number of RCHs for 64QAM modulation with coding rate 3/4 of LCH. The first graph of each figure shows the maximum throughput that it can achieve according to each modulation scheme. In that case only one active user and one $\mathrm{RCH}$ are used for simulation.

Note that the gap of throughput loss between two cases with and without guard time value becomes larger as we add the number of active users. This phenomenon is similar to that in Fig.8 and Fig.9.

\section{Conclusions}

In this paper we have discussed the effects of guard timing spaces in a MAC frame of ETSI BRAN HIPERLAN/2 system on the throughput performance. These guard timing spaces are needed between RCHs and different MAC-ID PDU trains in UL, and different sectors. We estimated throughputs under various guard time values defined in [2], the number of RCHs and number of DLCCs used by active users. We analyzed the required guard timing spaces in every PDU trains according to [1][2] and calculated their lengths in a number of OFDM symbol imposed on MAC frame depending on the number of RCHs and the number of DLCCs. The numerical results showed that the required length for the sectorized system is at most 13 OFDM symbols except for maximum guard time value, and that is relatively insignificant comparing to those for multi-DLCC of UL PDU trains and multi-RCH. It is obvious that 
the throughputs of system are dramatically dropped as the guard time value becomes bigger. The system capacity may be increased by decreasing the guard time value between the UL PDU trains and the RCHs, and infrequent use of SCHs. Also we showed by numerical results that it should be carefully estimated by AP/CC to determine the number of RCHs and the number of DLCCs of UL PDU trains because they are directly proportional to the length of required guard timing spaces and may, otherwise, degrade the performance of the system.

\section{References}

1. HIPERLAN Type2; Data Link Control(DLC) Layer; Part1: Basic Data Transport Functions, Broadband Radio Access Networks(BRAN), ETSI TS 101 761-1 V1.3.1, Dec(2001).

2. HIPERLAN Type2; Physical(PHY) Layer: Broadband Radio Access Networks(BRAN), ETSI TS 101475 V1.3.1, Dec(2001).

3. HIPERLAN Type2; Packet based Convergence Layer; Part1: Common Part, Broadband Radio Access Networks(BRAN), ETSI TS 101 493-1 V1.1.1, Apr.(2000).

4. Bernhard H. WALKE, et al. "IP over Wireless Mobile ATM-Guaranteed Wireless QoS by HiperLAN/2", Proc. IEEE, vol.89, no.1, Jan. (2001).

5. Andreas Hettich, Arndt Kadelka, "Performance Evaluation of the MAC Protocol of the ETSI BRAN HIPERLAN/2 Standard”, Proc. European. Wireless'99, Munich Germany, Oct. (1999).

6. Andreas Hettich, Matthias Schrother, "IEEE 802.11 or ETSI BRAN HIPERLAN/2: Who will win the race for a high speed wireless wireless LAN standard", Proc. European. Wireless'99, Munich Germany, Oct. (1999). 